\title{
Demonstration of a cavity-based pulse compression system for pulse shape correction
}

\author{
Yuliang Jiang, Hao Zha, Ping Wang, Jiaru Shi, ${ }^{*}$ and Huaibi Chen \\ Department of Engineering Physics, Tsinghua University, Beijing CN-100084, China \\ and Key Laboratory of Particle and Radiation Imaging, Tsinghua University, \\ Ministry of Education, Beijing CN-100084, China \\ William L Millar ${ }^{\dagger}$ and Igor Syratchev \\ The European Organization for Nuclear Research, Geneva CH-1211, Switzerland
}

(Received 28 May 2019; published 7 August 2019)

\begin{abstract}
A compact X-band correction cavity chain (CC) has been successfully designed, fabricated and high power tested for the Compact Linear Collider (CLIC) project. The CC consists of eight spherical cavities and four polarizers, and each spherical cavity has a $Q_{0}$ of 45000 and a diameter of $7 \mathrm{~cm}$. The compact $\mathrm{CC}$ can correct a decaying pulse shape generated by a storage cavity without need of low efficiency pulse modulation or a solution based on long delay lines. A flattop output with small ripples can be obtained if the $\mathrm{CC}$ and the storage cavity are matched. Experiments of the $\mathrm{CC}$ with a barrel open cavity (BOC) were conducted on the XBOX2 test facility at CERN, and the high power results match the theoretical calculations. The appliance of the $\mathrm{CC}$ only reduces the pulse compression efficiency by $6 \%$ relatively if the transmission attenuation is ignored.
\end{abstract}

DOI: 10.1103/PhysRevAccelBeams.22.082001

\section{INTRODUCTION}

The pulse compressor stores energy of the forepart pulse and releases it together with the remaining part under certain conditions [1-3]. The long input pulse is compressed to a short one with much higher amplitude. Commercial power sources generate long pulses at moderate power for optimal cost-efficiency [4-6]. Therefore, to achieve higher energy, pulse compressors have been key components and widely applied in large accelerator facilities [7-10]. Most passive pulse compressors emit out power after reversing the phase of rf pulses at a certain time [1], and they are the most used and developed types due to simple structures.

SLAC Energy Doubler (SLED) was invented in 1974 as the first pulse compressor. Conventional SLED pulse compressor consists of two resonant cavities and a $3-\mathrm{dB}$ coupler $[1,11,12]$. Over the past forty years, pulse compressors with resonant cavities have been developed. A barrel open cavity (BOC) uses a whispering gallery

\footnotetext{
shij@tsinghua.edu.edu.

${ }^{\dagger}$ Also at Cockcroft Institute, Lancaster University, Lancaster LA1 4YW, United Kingdom.

Published by the American Physical Society under the terms of the Creative Commons Attribution 4.0 International license. Further distribution of this work must maintain attribution to the author(s) and the published article's title, journal citation, and DOI.
}

mode operating in a resonant rotating wave regime, where a single cavity is sufficient for pulse compression [13-15]. In 2016, a compact SLED pulse compressor utilizing a single spherical cavity and a rf polarizer was invented. This design has been successfully applied in X-band, C-band, and S-band [16-18].

Even though cavity-based pulse compressors like SLED and BOC enhance the peak power, they output exponentially decaying pulses. However, specific pulse shapes, especially the flattop, are required in some situations. For the Compact Linear Collider (CLIC) hosted by CERN, its collision energy is designed to be built and increased in three stages, which are $380 \mathrm{GeV}, 1.5 \mathrm{TeV}$ and $3 \mathrm{TeV}$ [19]. For the first stage, powering schemes based on two-beam acceleration and high-efficiency X-band klystrons are both considered. The flattop pulse compression is essential in the klystron-based scheme.

Phase and amplitude modulations can correct the decaying pulse shape at the expense of lowering the power gain and the pulse compression efficiency. Furthermore, the modulated output phase isn't stable for a single input [20]. To avoid the additional inefficiency due to pulse shape, binary power multiplier (BPM) and SLED-II systems directly generate flattop pulses with delay lines [21-23]. But the delay line systems are impractical at X-band and other lower frequencies, since the delay line length is proportional to the pulse length. Thus, a compact system that corrects the pulse shape with a considerable pulse compression efficiency is needed. 


\section{DESIGN OF CORRECTION CAVITY CHAIN}

The SLED and SLED-II systems store energy in resonant cavities and delay lines respectively. In fact, theories of SLED and SLED-II are the same phenomenon described in different regimes of resonator dimensions and coupling [24]. The operating mode of the delay line has a higher longitudinal index, and the spectrum is more dense around the operating frequency. This means the spectrum with harmonics responses to a flat pulse more faithfully. Therefore, a cavity-based system can partly function like a delay line as an alternative. The idea is to put several resonant peaks around the operating frequency to simulate the delay line, which can be realized by coupled cavities or a cavity chain [25-27]. Such a system is expected to correct the pulse shape generated by a SLED or BOC type pulse compressor without need of low efficiency pulse modulation $[20,28]$ or a solution based on long delay lines.

As a linear system, the frequency spectrum of a given input pulse can be produced by taking Fourier Transform. Then the time domain output can be obtained by taking inverse Fourier transform of the frequency domain product of the input and the system response. Therefore, simulations and optimizations of a cavity-based system for a desired pulse shape can be realized by codes based on fast Fourier transform (FFT).

In the simulation, resonant cavities are characterized by the resonant frequency $(f)$, the unloaded quality factor $\left(Q_{0}\right)$ and the coupling coefficient $(\beta)$. These three parameters are optimized based on the following ideas. As for the resonant frequencies $(f)$ for a flattop output pulse, their values can refer to the delay line performance, where the traveling waves are superimposed with a fundamental delay time of the round-trip. Therefore, the frequency difference of two neighboring peaks is set to be the reciprocal of the output pulse length. This equation is also implicit in Fourier transform. As for the unloaded factor $\left(Q_{0}\right)$ and the coupling coefficient $(\beta)$, unlike the storage cavity, correction cavities are not tuned at the resonant frequency and contain little rf energy. Thus, unloaded quality factors $\left(Q_{0}\right)$ of correction cavities can be comparatively smaller in the slightly over coupled case $(1<\beta<2)$.

In recent years, we have studied, designed and fabricated a correction cavity chain (CC) to demonstrate the idea. The $\mathrm{CC}$ consists of four units, and each unit is composed of two coupled spherical cavities and a dual-mode polarizer, as shown in Fig. 1. The unloaded quality factor $\left(Q_{0}\right)$ of each spherical cavity is around $4.5 \mathrm{e} 4$, with a coupling coefficient $(\beta)$ of about 1.5. The polarizer excites two polarized modes azimuthal in both space and time to direct the power flow. A 0 -like and a $\pi / 2$-like modes are excited in each of the four coupled cavity pairs. Eight peaks are generated around the operating frequency $(11.994 \mathrm{GHz})$, and the frequency difference between two nearby peaks is $4 \mathrm{MHz}$. Therefore, the CC corrects a $250 \mathrm{~ns}$ output pulse (for details refer to Ref. [27]).

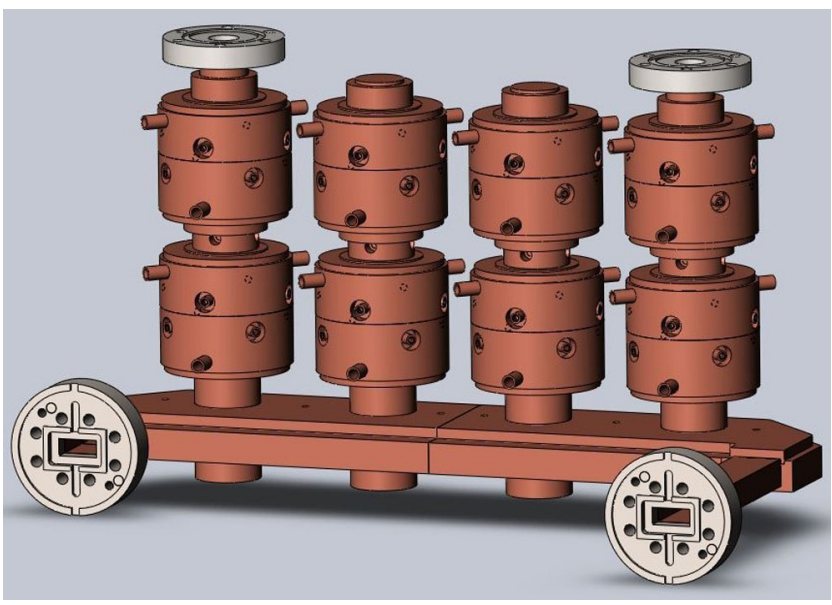

FIG. 1. The mechanical design of the CC.

Currently, the SLEDX [29] and the BOC [30] are available as a storage cavity at $11.994 \mathrm{GHz}$. The corrected pulse shapes of the SLEDX and the BOC are shown in Fig. 2(a),(b) respectively. The BOC has a lower $Q_{0}$ and a higher $\beta$ than the SLEDX. Therefore, the power inside BOC decays more quickly after the phase reversal, which this CC can't fully compensate. For straight comparisons, the flattop outputs for a SLEDX, a BOC, and a BOC-CC system after the amplitude modulation (AM) are shown in Fig. 2(c),(d),(e) respectively. The CC corrects the pulse shape with a higher power gain while the AM realizes it by lowering down the input power.
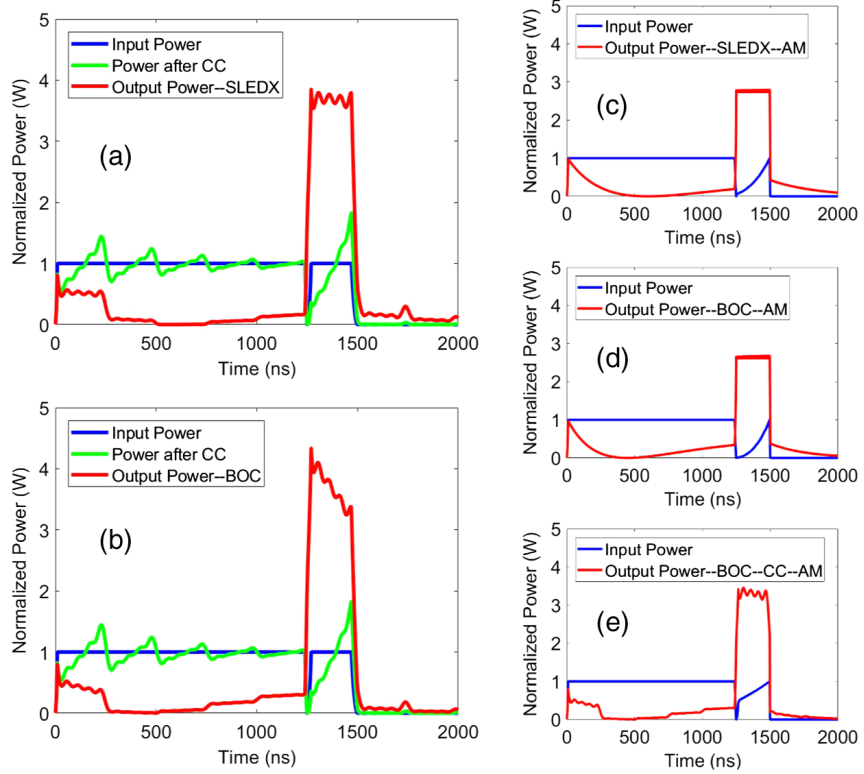

FIG. 2. (a): The corrected waveforms of the $\operatorname{SLEDX}\left(Q_{0}=\right.$ $177000, \beta=5.98$ ). (b): The corrected waveforms of the BOC $\left(Q_{0}=158000, \beta=7\right)$. (c): The amplitude-modulated(AM) waveforms of the SLEDX. (d) The AM waveforms of the BOC. (e) The AM waveforms of the BOC-CC system. All the waveforms are simulated based on theoretical characterization. 
Thus, a flattop output with small ripples can be obtained if the CC and the storage cavity are matched. Even in the unmatched case, the CC can increase the power gain after the amplitude modulation (AM).

\section{MICROWAVE MEASUREMENTS}

The CC components were assembled for measurements and tuning, as shown in Fig. 3. Since two cavities are coupled and the system is operated at a polarization mode, tunings of the absolute cavity frequency and of the frequency difference between cavities were implemented in turns. Using tuning holes on the cavities at different angles, the cavity frequency was tuned while equaling frequencies of the polarization mode. Using the tuning holes on the small cylindrical cavity in the middle, the frequency difference between two coupled cavities was tuned. The frequency spectrum after tuning is shown in Fig. 4.

For the whole pulse compression system, a BOC cavity developed by PSI was adopted as the storage cavity. The whole pulse compression system was measured in CERN before high power tests, as shown in Fig. 5. Two chillers and an ion pump were equipped for an accurate spectrum. The BOC frequency was set right in the middle of two nearby resonant peaks for the best performance. The spectrums of the system are shown in Fig. 6.

According to the spectrums of the $\mathrm{CC}$ and the whole system, both the CC and the BOC were well tuned, but there is a larger transmission attenuation than expected. The transmission coefficients of the CC and the whole system including connecting waveguides are $-0.46 \mathrm{~dB}$ and $-0.93 \mathrm{~dB}$ respectively. Therefore, both the BOC and the $\mathrm{CC}$ have a transmission efficiency of 0.9. The transmission attenuation of the CC mainly comes from the four polarizers. However, simulation results of the CC polarizers only show an transmission coefficient of $-0.12 \mathrm{~dB}$,

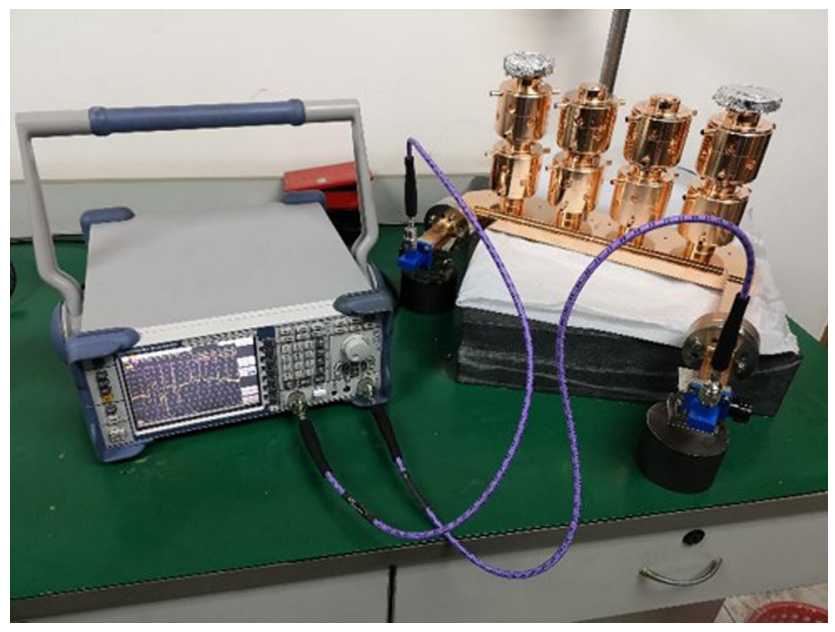

FIG. 3. Microwave measurement of the $\mathrm{CC}$ during tuning.

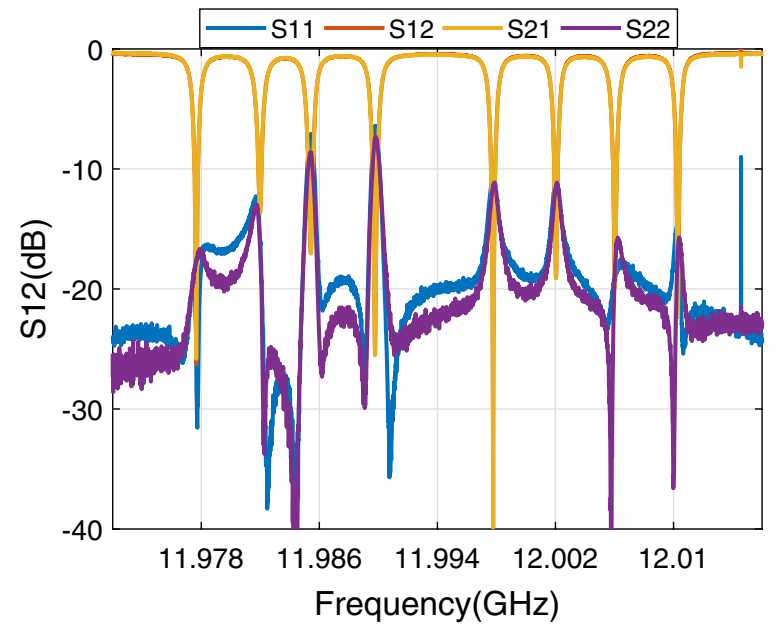

FIG. 4. The frequency spectrum of the $\mathrm{CC}$ after tuning. The transmission coefficient of the $\mathrm{CC}$ is $-0.46 \mathrm{~dB}$.

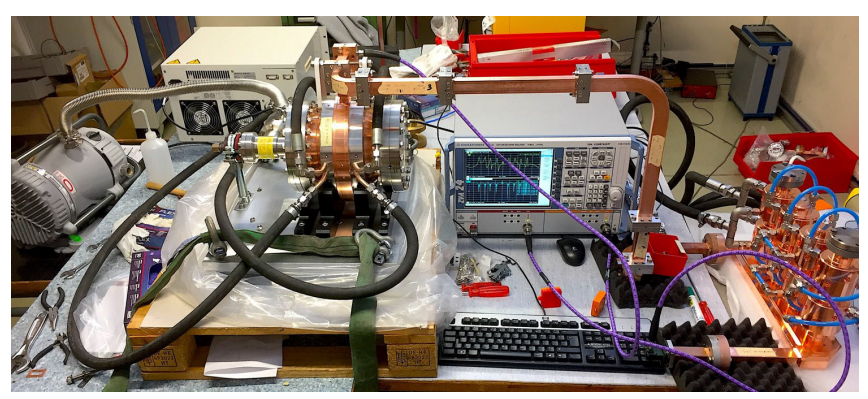

FIG. 5. Measurements of the pulse compression system.

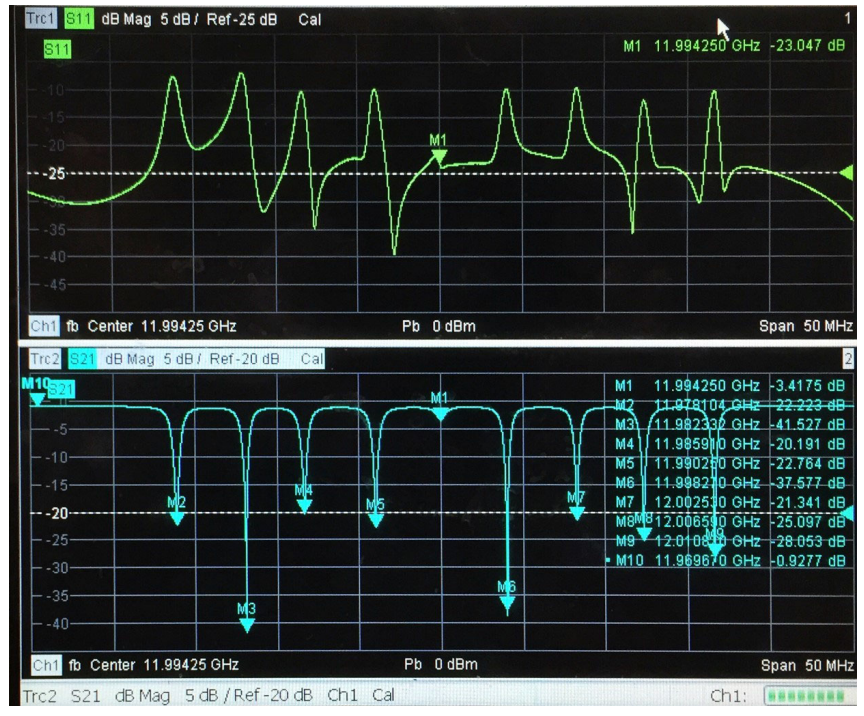

FIG. 6. The spectrums of the pulse compression system including the $\mathrm{CC}$, the $\mathrm{BOC}$ and the connecting waveguides.

corresponding to a transmission efficiency of about 0.97 . The actual surface roughness of components possibly accounts for the extra attenuation, which can be perfected next time. 


\section{HIGH POWER TESTS}

The pulse compression system was high-power tested on the XBOX2 test facility at CERN, as shown in Fig. 7. The simplified rf layout of XBOX2 is illustrated in Fig. 8. The 50 MW XL5 based klystron modulator system generates pulsed power with a pulse width and repetition rate of $1.5 \mu \mathrm{s}$ and $50 \mathrm{~Hz}$ respectively [12]. The CC and the BOC are tested to modulate and compress the klystron output pulse. The enhanced power is then fed into a testing structure and two rf loads. High power tests were conducted in two stages. The first stage conditioned the new installed $\mathrm{CC}, \mathrm{BOC}$ and a rf load with no structure under test. In the second stage, a deflecting structure called Polarix [31] was installed and tested simultaneously.

In the high power tests, the pulse length is $1.5 \mu \mathrm{s}$, with a 180-degree phase implemented $250 \mathrm{~ns}$ before the end of the pulse. The output peak power is $20.8 \mathrm{MW}$, and the peak power gain is about 3.5 as the average input power is about 6 MW. Few breakdowns have happened in the CC.

The waveforms for the high power tests in comparison with the simulated calculations considering transmission attenuation are shown in Fig. 9(a),(b). Pulse shapes corrected by the $\mathrm{CC}$ match the theoretical design, although calibration errors and fluctuations of the chiller temperatures result in minor difference at the power level and the ripples of the output respectively. This fully demonstrates the idea of utilizing a cavity chain for pulse shape correction. Thus, a flattop pulse is reasonable if a SLEDX is used or the BOC is equipped with a CC of a larger $Q_{l}$.

To analyze the transmission attenuation and the pulse compression efficiency better, the simulations of the system and a single BOC without attenuation are shown in Fig. 9 (c),(d). The transmission spectrum of the system without attenuation is achieved by moving the base line from the measured $-0.93 \mathrm{~dB}$ to the ideal $0 \mathrm{~dB}$, and the spectrum of a single $\mathrm{BOC}$ is generated according to its $Q_{0}$ and $\beta$. The calculated results are listed in Table I.

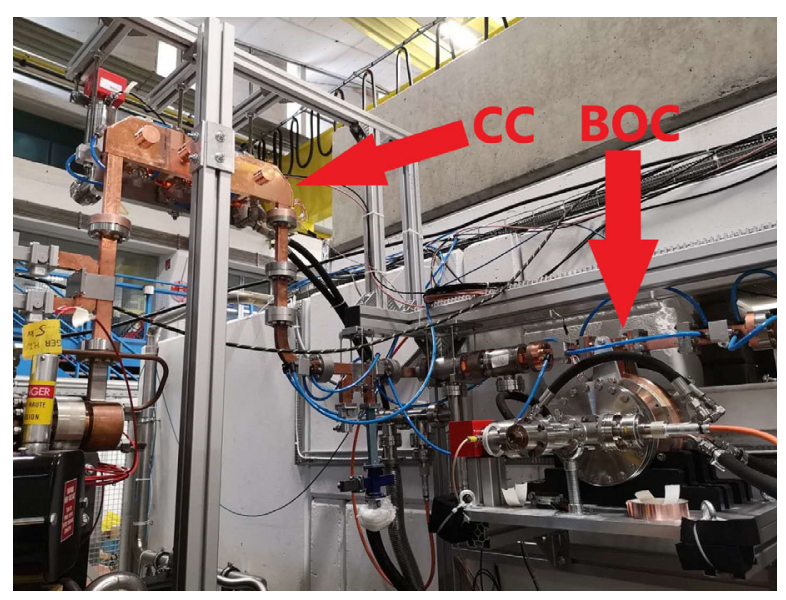

FIG. 7. Installation of the pulse compression system.

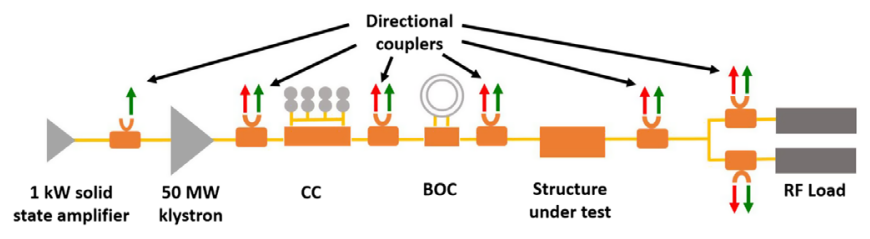

FIG. 8. XBOX2 block diagram.
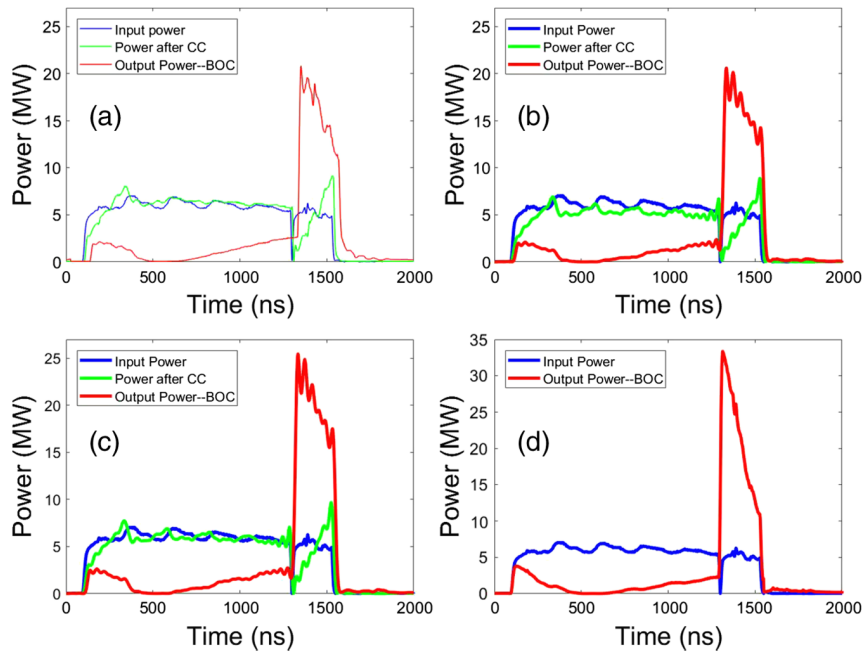

FIG. 9. (a) The measured waveforms. (b) The calculated waveforms with attenuation. (c) The calculated waveforms without attenuation. (d) The calculated waveforms of a single BOC without attenuation.

Both the CC and the BOC attenuate the power by $10 \%$ during transmission, thus the pulse compression efficiency reduces from $57 \%$ to $46 \%$ if considering the attenuation. In the condition of no attenuation, the efficiency is only reduced by $6 \%$ relatively if the $\mathrm{CC}$ is equipped. It is reasonable to believe that after perfecting the polarizers'

TABLE I. The calculated results according to waveforms.

\begin{tabular}{|c|c|c|c|c|}
\hline Types $^{a}$ & $\begin{array}{l}\text { Peak } \\
\text { power } \\
(\mathrm{MW})\end{array}$ & $\begin{array}{c}\text { Initial power } \\
\text { after CC } \\
(\mathrm{MW})\end{array}$ & $\begin{array}{c}\text { Initial } \\
\text { power } \\
\text { after BOC } \\
(\mathrm{MW})\end{array}$ & $\begin{array}{c}\text { Pulse } \\
\text { compression } \\
\text { efficiency } \\
(\%)\end{array}$ \\
\hline $\begin{array}{l}\text { The measured } \\
\text { system }\end{array}$ & 20.8 & 2.8 & 1.9 & 45.0 \\
\hline $\begin{array}{l}\text { The designed } \\
\text { system w/ } \\
\text { attenuation }\end{array}$ & 20.6 & 2.7 & 2.0 & 45.8 \\
\hline $\begin{array}{c}\text { The designed } \\
\text { system w/o } \\
\text { attenuation }\end{array}$ & 25.5 & 3.1 & 2.5 & 56.8 \\
\hline $\begin{array}{l}\text { A single BOC } \\
\text { w/o attenuation }\end{array}$ & 33.4 & I & I & 60.7 \\
\hline
\end{tabular}

${ }_{\mathrm{a}}^{\mathrm{a}} \mathrm{/} / \mathrm{=}$ with, w/o = with out.

${ }^{\mathrm{b}}$ The initial power means the output power after the $\mathrm{CC}$ or the BOC when the input is on. 


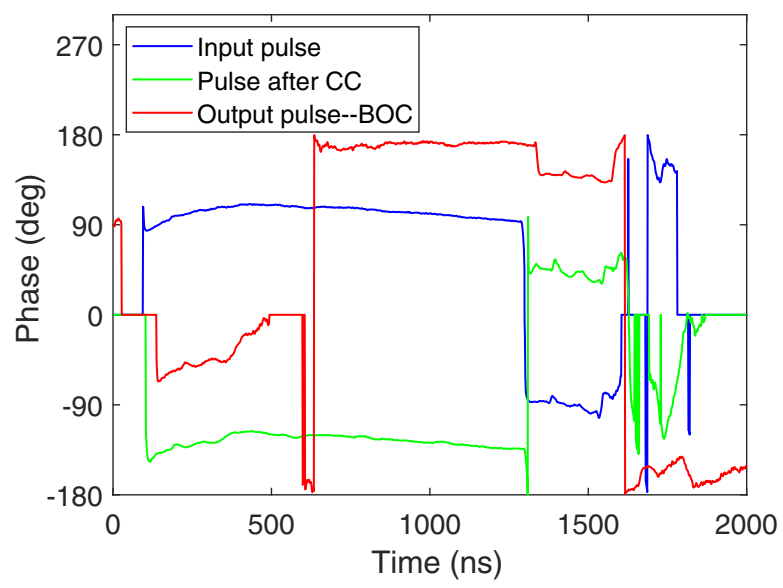

FIG. 10. Phase information.

surface roughness, the compact $\mathrm{CC}$ will correct the pulse shape with a higher power gain and considerable efficiency in the future applications.

Phases of the pulse after the CC and the output pulse responses to the input phase stably, as shown in Fig. 10. The fluctuations of phases mainly results from the input pulse phase after the phase reversal, and little phase instability is introduced to the system by the CC.

\section{CONCLUSION}

This work successfully demonstrates the idea of the compact CC for pulse shape corrections. The power gain and the pulse compression efficiency could be higher if the surface roughness is improved. The $\mathrm{CC}$ can also be scaled and applied in C-band and S-band easily.

\section{ACKNOWLEDGMENTS}

Special thanks to Matteo Volpi for his contribution and expertise in operating the experimental setup at the CERN XBOX2. The authors are grateful to Walter Wuensch, Nuria Catalan Lasheras, and Alexej Grudiev for useful discussions and Riccardo Zennaro for leading the ARIES TA programme. This project was supported by the National Natural Science Foundation of China (No. 11635006). This project has received funding from the European Union's Horizon 2020 Research and Innovation programme under Grant Agreement No. 730871.

[1] Z. Farkas, H. Hoag, P. B. Wilson, and G. Loew, Sled: A method of doubling slac's energy, in Proc. of 9th Int. Conf. on High Energy Accelerators, Stanford (1974), p. 576, http://inspirehep.net/record/94052/files/HEACC74_597604.pdf.

[2] S. G. Tantawi, R. D. Ruth, and A. E. Vlieks, Active radio frequency pulse compression using switched resonant delay lines, Nucl. Instrum. Methods Phys. Res., Sect. A 370, 297 (1996).

[3] O. A. Ivanov, V. A. Isaev, M. A. Lobaev, A. L. Vikharev, and J.L. Hirshfield, A resonance switch employing an explosive-emission cathode for high-power rf pulse compressors, Appl. Phys. Lett. 97, 031501 (2010).

[4] S. H. Gold and G. S. Nusinovich, Review of high-power microwave source research, Rev. Sci. Instrum. 68, 3945 (1997).

[5] S. Choroba, J. Hameister, S. Jarylkapov et al., Performance of an s-band klystron at an output power of $200 \mathrm{mw}$, in Proceedings of the 19th International Linear Accelerators Conference, Chicago, IL, 1998 (NTIS, Springfield, VA, 1998), pp. 917-919.

[6] D. Sprehn, G. Caryotakis, A. Haase, E. Jongewaard, and C. Pearson, Current status of the next linear collider x-band klystron development program, in Proceedings of the 9th European Particle Accelerator Conference, Lucerne, 2004 (EPS-AG, Lucerne, 2004), pp. 1090-1092 [http://accelconf .web.cern.ch/AccelConf/e04/].

[7] R. Akre, V. Bharadwaj, P. Emma, and P. Krejcik, Slac linac rf performance for lcls, physics/0008171.

[8] T. Schietinger, M. Pedrozzi, M. Aiba et al., Commissioning experience and beam physics measurements at the swissfel injector test facility, Phys. Rev. Accel. Beams 19, 100702 (2016).

[9] T. Inagaki, C. Kondo, H. Maesaka, T. Ohshima, Y. Otake, T. Sakurai, K. Shirasawa, and T. Shintake, High-gradient $c$-band linac for a compact x-ray free-electron laser facility, Phys. Rev. Accel. Beams 17, 080702 (2014).

[10] Y. Joo, H.-S. Lee, W. Hwang, Y. Park, K. Oh, and B.-J. Lee, Design study of a new sled system with a biplanar 3 -db power divider and dual side-wall coupling-irises for the pal xfel, J. Korean Phys. Soc. 63, 1253 (2013).

[11] H. Matsumoto, H. Baba, S. Yamaguchi, and A. Miura, High power test of a sled system with dual side-wall coupling irises for linear colliders, Nucl. Instrum. Methods Phys. Res., Sect. A 330, 1 (1993).

[12] B. Woolley, I. Syratchev, and A. Dexter, Control and performance improvements of a pulse compressor in use for testing accelerating structures at high power, Phys. Rev. Accel. Beams 20, 101001 (2017).

[13] I. Syrachev, V. Vogel, H. Mizuno, J. Odagiri, Y. Otake, S. Tokumoto et al., The results of if high power tests of $\mathrm{x}$-band open cavity rf pulse compression system vpm (jlc), in Proceedings of the 17th International Linear Accelerator Conference (LINAC-1994), Tsukuba, Japan, 1994 (KEK, Tsukuba, Japan, 1994), pp. 475-477.

[14] J. Hirshfield, S. V. Kuzikov, M. I. Petelin, and V. G. Pavelyev, Whispering gallery pulse compressor, AIP Conf. Proc. 737, 637 (2004).

[15] R. Zennaro, M. Bopp, A. Citterio, R. Reiser, and T. Stapf, C-band rf pulse compressor for swissfel, in 4th Int. Particle Accelerator Conf.(IPAC'13), Shanghai, China (JACOW Publishing, Geneva, Switzerland, 2013), pp. 2827-2829.

[16] J. W. Wang, S. G. Tantawi, C. Xu, M. Franzi, P. Krejcik, G. Bowden, S. Condamoor, Y. Ding, V. Dolgashev, J. Eichner, A. Haase, J. R. Lewandowski, and L. Xiao, Development for a supercompact $x$-band pulse compression system and 
its application at slac, Phys. Rev. Accel. Beams 20, 110401 (2017).

[17] Z.-B. Li, W.-C. Fang, Q. Gu, J.-H. Tan, X.-X. Huang, and Z.-T. Zhao, Design, fabrication, and cold-test results of c-band spherical rf pulse compressor prototype, Radiation Detection Technology and Methods 3, 21 (2019).

[18] P. Wang, J. Shi, H. Zha, D. Cao, M. Peng, Z. Liu, C. Cheng, and H. Chen, Development of an s-band spherical pulse compressor, Nucl. Instrum. Methods Phys. Res., Sect. A 901, 84 (2018).

[19] T. CLIC, M. Boland, U. Felzmann, P. Giansiracusa, T. Lucas, R. Rassool, C. Balazs, T. Charles, K. Afanaciev, I. Emeliantchik et al., Updated baseline for a staged compact linear collider, arXiv:1608.07537.

[20] A. Fiebig and C. Schieblich, A SLED type pulse compressor with rectangular pulse shape, CERN Technical Report No. CM-P00059378, 1990.

[21] Z. Farkas, Binary peak power multiplier and its application to linear accelerator design, IEEE Trans. Microwave Theory Techn. 34, 1036 (1986).

[22] P. B. Wilson, Z. Farkas, and R. D. Ruth, SLED II: A new method of RF pulse compression, Stanford Linear Accelerator Center Technical Report, 1990.

[23] S. G. Tantawi, Multimoded reflective delay lines and their application to resonant delay line rf pulse compression systems, Phys. Rev. Accel. Beams 7, 032001 (2004).
[24] C. D. Nantista, Radiofrequency pulse compression for linear accelerators, Technical Report No. SLAC-R-95455, 1995.

[25] T. Shintake and N. Akasaka, A new RF pulse-compressor using multi-cell coupled-cavity system, KEK Technical Report No. SCAN-9609133, 1996.

[26] S. Y. Kazakov, Pulse shape correction for rf pulse compression system, Spectrum 5, 9 (1992).

[27] P. Wang, H. Zha, I. Syratchev, J. Shi, and H. Chen, rf design of a pulse compressor with correction cavity chain for klystron-based compact linear collider, Phys. Rev. Accel. Beams 20, 112001 (2017).

[28] A. Rezaeizadeh, R. Kalt, T. Schilcher, and R. S. Smith, An iterative learning control approach for radio frequency pulse compressor amplitude and phase modulation, IEEE Trans. Nucl. Sci. 63, 842 (2016).

[29] B. Woolley, High power X-band RF test stand development and high power testing of the CLIC crab cavity, Ph.D. thesis, Lancaster University (2015).

[30] R. Zennaro (private communication).

[31] P. Craievich, R. Aßmann, M. Bopp, H.-H. Braun, N. Catalán Lasheras, F. Christie, R. D’Arcy, U. Dorda, M. Foese, R. Ganter et al., Status of the polarix-tds project, in 9th Int. Particle Accelerator Conf.(IPAC'18), Vancouver, BC, Canada, April 29-May 4, 2018 (JACOW Publishing, Geneva, Switzerland, 2018), pp. 3808-3811. 